\title{
THE BRAKING LAW OF SOLAR TYPE STARS AS DERIVED FROM CLOSE BINARY DYNAMICAL EVOLUTION
}

\author{
CARLA MACERONI \\ Osservatorio Astronomico di Roma, I-00040 Monteporzio, Italy
}

\section{THE DYNAMICAL EVOLUTION OF CLOSE SOLAR TYPE BINARIES}

The orbital period of a close binary system $(P \sim$ a few days $)$, formed by two solar type components, evolves under the influence of angular momentum loss (AML) by electromagnetic braking and of spin $\leftrightarrow$ orbit angular momentum transfer (AMT) by tidal coupling. Because of AMT, which tends to reestablish the spin-orbit synchronization destroyed by AML, the loss of AM takes finally place at the expenses of the orbital reservoir and produces shrinking of the orbit and spinning-up of the components. The variation with time of $\omega_{K}$ and $\omega$, respectively the orbital and the rotational angular velocity, is expressed by a system of (stiff) ordinary differential equations and the results of the (numerical) integration strongly depend on the AML and AMT laws. A series of recent papers (Maceroni and Van 't Veer 1991, 1992, Van't Veer and Maceroni 1992, hereafter MV1, MV2 and VM) presents the resulting orbital period evolution function (PEF) with a variety of choices. A typical result, in terms of orbital period, and for a binary formed by two identical G5 V components, is shown in fig. IA. The solution shown here corresponds to the braking law a) of fig. IB , to the AMT according to Zahn (1977) and to stars rotating as rigid bodies; different assumptions have been tried in MV1and VM.

By means of PEFs similar to that of fig. IA, we computed the evolution of a given initial period distribution (IPD) into the present period distribution, PPD. This was compared with the observed distribution, after correction for detectability and selection effects. The comparison constraints the AML and AMT relations by the requirement of reproducing two remarkable observed properties of close binaries : the bimodality of the PPD of G type binaries, with a dip at short periods $0.2<\log P<0.4$, (Farinella et al., 1979) and their almost perfect spin-orbit synchronization (Tan et al., 1991), with a value of the synchronization parameter $f=\omega / \omega_{K}>0.96$ for $P<10^{d}$. In the computations we assumed a time-independent IPD, following a flat $N(\log P)$ distribution, (Abt 1983), and an age of the galactic disk of $\sim 810^{9} \mathrm{y}$. The results can be summarized as follows:

- The bimodal distribution of $\mathrm{G}$ type binaries cannot be reproduced with a braking law following the Skumanich relation on the whole rotational range. All the distributions computed with this assumption are increasing functions of $\log P$ (Fig. II b).

- The dip at $\log P \approx 0.3$ requires a braking law for fast rotators $\left(\omega \geq 12 \omega_{\odot}\right)$ independent from $\omega$. The amount of this $\omega$-independent braking, and its range of validity, imply a rapid increase of $|\dot{\omega}|, \Delta \log |\dot{\omega}| \simeq 1.5$ for 

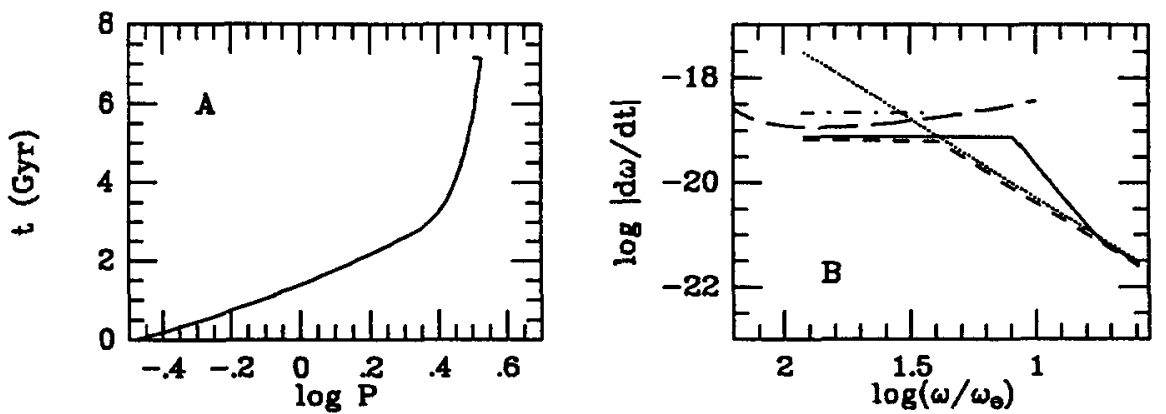

FIGURE I A) The orbital period evolution function (PEF). The time is counted backwards from contact time $(t=0)$. B) Three braking laws corresponding to the PPD of fig. II. a) continuous line: the Skumanich $\left(\omega \propto t^{-1 / 2}\right)$ relation for slow rotators $+a \log |\dot{\omega}|=$ const for fast rotators, $+a$ tentative connection between the two. This relation yields the best fit of the observed distributions, b) dotted line: pure Skumanich relation, extrapolating that of slow rotators, $c$ ) dashed line: the same Skumanich relation, extrapolated to the intersection with the $\log |\dot{\omega}|=$ const line + this $\omega$-independent law. Dashed-dotted line: braking computed from the data of $\mathrm{G}$ type stars in $\alpha$ Per and Pleiades. Long dashed line: spin-down model of Schatzman and Tutukov (1992)

$6<\omega / \omega_{\odot}<12$ (fig. IB, law a, , fig. II a).

The rate of spin-down at intermediate $\omega$ 's (i.e. $12<\omega / \omega_{\odot}<25$ is much higher than that predictable by extrapolation of the Skumanich relation of single stars (Soderblom, 1983). The lower $\omega$ boundary $\left(\omega_{1}\right)$ of this fast rotator relation is well constrained by the dip location and the requirement $f>0.96$. Lower values yield de-synchronized systems, because of the lower efficiency of the synchronization mechanism $\left(\propto \omega^{4}\right)$, higher values shift the dip to too short periods. For instance, a relation made out of a Skumanich law, extrapolated towards higher $\omega$ 's to the intersection with the $\dot{\omega}=$ const section and, from there on, by that constant value (short dashed line of fig. IB) is ruled out by its PPD shape (fig. II c).

\section{DISCUSSION}

If binarity and tidal coupling do not affect the AML process by changing, for instance, the magnetic field structure or the mass loss rate, as the observations of the surface activity phenomena in binaries seem to indicate (Young et al., 1989), the results of binaries can be compared with those of single stars. Therefore, In fig. IB, we compare our best braking law with a rough estimate of the spindown of fast rotators from the data for galactic clusters (Stauffer 1991) and with a theoretical curve based on the model of spin-down of Schatzman and Tutukov (1992). In this model the rapid increase of the spin-down rate, in spite of the reduced braking efficiency with increasing rotation of the magnetic field, is explained in terms of a change in the mass loss rate, due to the transition 

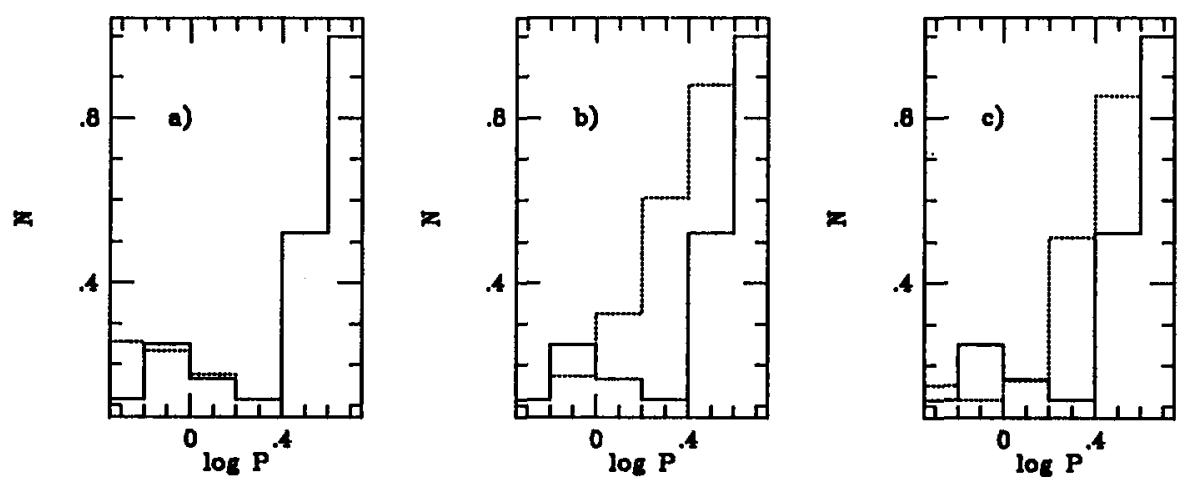

FIGURE II Comparison of the computed (dotted line) present period distribution (PPD) with the observed (corrected) one (continuous line). The three figures correspond to the three braking laws of fig. IB

from thermal or Alfvenic to centrifugal wind. This transition should take place at $\omega \sim 11 \omega_{\odot}$, in good agreement with our results.

The data of young fast rotators suggest that the braking is initially acting only on the external (convective) layers, while what we know on the solar rotation suggests an almost rigid body rotation at later times (and slower rotation). We should therefore include in our models a rotation dependent core-envelope coupling. If this trend of coupling with rotation applies to binaries as well, the smaller inertial moment of the involved layers of fast rotators would produce an upward shift of the fast rotator relation. That qualitatively goes in the sense of improving the agreement between our results and those for single stars.

\section{REFERENCES}

Abt, H.A., 1983, ARA\&A, 21, 343

Farinella, P., Luzny, F., Mantegazza, L., Paolicchi, P., 1979, ApJ, 234, 973

Maceroni, C., Van 't Veer, F., 1991, $A \mathcal{E} A$, 246, 91

Maceroni, C., Van 't Veer, F., 1992, in preparation

Schatzman, E., Tutukov, A., 1992, to be published on $A \mathcal{E} A$

Soderblom, D. R., 1983, ApJS, 53, 1

Stauffer, J. R., 1991 in “ Angular Momentum Evolution of Young stars”, S. Catalano and R.J. Stauffer eds., Kluwer Acad. Publ.

Tan, H., Wang, X., Pan, K., 1991, Acta Astron. Sin. 32/2, 145

Van 't Veer F., Maceroni C., 1992 in "Binaries as tracers of stellar formation",

Eds. A. Duquennoy and M. Mayor, Cambridge University press, in press

Young, A., Ajir, F., Thurman, G., 1989, PASP, 101, 1017

Zahn, J.P., 1977, $A \mathcal{E} A$, 57, 383 